Projets

de paysage

\section{Projets de paysage}

Revue scientifique sur la conception et l'aménagement de l'espace

$17 \mid 2017$

Paysage(s) et agriculture(s)

\title{
Voyage en terres urbaines cultivées
}

Au Reyerhof, à Stuttgart-Möhringen

Landwirtschaft auf stadtnahen Flächen - Erkundungen auf dem Reyerhof in Stuttgart-Möhringen

\section{Dominique Henry}

\section{(2) OpenEdition}

\section{Journals}

Édition électronique

URL : http://journals.openedition.org/paysage/4554

DOI : $10.4000 /$ paysage. 4554

ISSN : 1969-6124

Éditeur :

École nationale supérieure du paysage de Versailles-Marseille, Institut national des sciences appliquées Centre Val de Loire - École de la nature et du paysage, École nationale supérieure d'architecture et de paysage de Bordeaux, École nationale supérieure d'architecture et de paysage de Lille, Agrocampus Angers

Référence électronique

Dominique Henry, «Voyage en terres urbaines cultivées », Projets de paysage [En ligne], 17 | 2017, mis en ligne le , consulté le 03 avril 2020. URL : http://journals.openedition.org/paysage/4554; DOI : https://doi.org/10.4000/paysage.4554

Ce document a été généré automatiquement le 3 avril 2020.

Projets de paysage 


\title{
Voyage en terres urbaines cultivées
}

\author{
Au Reyerhof, à Stuttgart-Möhringen \\ Landwirtschaft auf stadtnahen Flächen - Erkundungen auf dem Reyerhof in \\ Stuttgart-Möhringen
}

Dominique Henry

\section{NOTE DE L'AUTEUR}

Avec l'aimable participation de Lukas Dreyer, agriculteur et maraîcher, cogestionnaire du Reyerhof et d'Alina Reinartz, architecte-urbaniste et agricultrice, membre fondateur de la SoLaWis.

\section{«L'agriculture dans la ville, pour la ville »}

1 La ville et l'agriculture ont, toutes deux, besoin d'espace. C'est un fait. L'étendue est au cœur de leur déploiement. Seulement, le foncier à bâtir de l'une est le sol à cultiver pour l'autre. C'est un autre fait, l'expansion continue des villes procède donc par soustraction des terrains à l'agriculture (et à la forêt). Ainsi les villes enflent, s'étendent, s'élargissent mais aussi parfois entourent, enserrent, englobent les terres agricoles. Car l'urbanisation, en dehors de la ville dense des centres anciens, n'est pas une nappe continue et homogène - de constructions et d'infrastructures. C'est bien plutôt, généralement, un phénomène spatial à maille lâche, particulièrement dispendieux en terrain et générant des paysages que l'on peine souvent à décrire parfois à apprécier - avec nos grilles de lecture habituelles reposant encore largement sur l'idée d'un grand partage entre ce qui serait d'un côté la ville compacte et de l'autre la campagne agricole. Il en résulte un dessin toujours complexe de ces extensions urbaines dont les formes sont parfois décrites comme tentaculaires, comme les pièces d'un puzzle mal ajustées, comme un nuage de confettis, ou encore comme une surface lacunaire laissant subsister des « enclaves agricoles » (Janin, 2009). 
2 Cette "extension du domaine de l'urbanisme » (Masboungi, 2014) tend à créer une grande variété de contacts, de rapprochements, de voisinages nouveaux, négociés ou non entre la ville et son entour agricole. La plupart du temps, contraints, les agriculteurs sont obligés de céder la place, de reculer, sinon de s'adapter. Mais on voit aussi apparaître des initiatives, publiques et privées, qui cherchent à négocier, à anticiper et à stabiliser le partage de l'espace. C'est le cas, par exemple, de métropoles qui prennent en charge le ménagement des terres agricoles à travers les concepts de parc agricole en Toscane (Bernetti et al., 2008), de "champs urbains" à Rennes (Boutleux, 2013), ou d'associations tel le Triangle vert ${ }^{1}$ issu du regroupement des villes maraîchères du Hurepoix (Laverne, 2011). Moins connus que les projets des collectivités, on trouve aussi les cas d'agriculteurs qui cherchent à s'adapter, à tenter de faire front et d'affirmer leur nécessité. Leurs démarches consistent à tirer profit de leur situation urbaine et à élaborer un ensemble de rapprochements avec la ville. Résultant d'une histoire toujours singulière, leurs démarches, parsemées dans les territoires métropolitains, demanderaient à être identifiées et analysées, les paysages agricoles étudiés, et les agriculteurs comme les consommateurs concernés écoutés. Il s'agirait de mener une investigation sur le terrain des paysages et de mieux saisir cette notion de rapprochements qui semble éclore entre agriculteurs et villes. Sans doute serions-nous alors en mesure d'alimenter l'ambition d'un projet agricole pour la ville tel que le formule Rémi Janin dans son stimulant essai (Janin, 2017). Et cela pourrait former un horizon à de futures recherches paysagères, quand bien même des travaux abordent déjà en partie ce champ (Toublanc et Poulot, $2017^{2}$ par exemple).

3 L'objet de cette contribution est, plus modestement, de porter un regard sur les paysages agricoles fabriqués par une exploitation agricole au contact étroit d'une agglomération comme Stuttgart. Il s'agit du Reyerhof', une ferme en polycultureélevage biodynamique, dans laquelle j'ai régulièrement l'occasion de séjourner et de faire mes courses dans son magasin. C'est son slogan «L'agriculture dans la ville, pour la ville ${ }^{4}$ » qui m'a donné envie d'effectuer le voyage en (ses) terres urbaines cultivées, et d'en livrer ici un récit. Ce slogan, que l'on retrouve estampillé sur le fourgon de livraison et les publicités, traduit autant un argument commercial qu'un état de fait. Les parcelles travaillées par la ferme s'inscrivent en effet dans le territoire urbanisé de Stuttgart, elles occupent la lisière des quartiers et s'intercalent plus spécifiquement entre Möhringen et les autres communes qui organisent la partie sud de cette agglomération. Habitant occasionnel de la ferme et consommateur de ses produits, l'envie m'est donc venue de connaître les lieux qui la composent et desquels proviennent les légumes dont je suis amateur.

4 L'idée du voyage ${ }^{5}$ ici développée exprime celle de vouloir faire la rencontre ${ }^{6}$, à travers un cheminement pédestre, des paysages agricoles et de ceux qui les cultivent. Il s'agit de Lukas, agriculteur et maraîcher, cogestionnaire du Reyerhof avec qui je me suis entretenu, et de Alina d'abord architecte puis agricultrice, salariée depuis peu par la ferme après y avoir effectué un apprentissage, et membre fondatrice de la SoLaWi de Stuttgart $^{7}$. Par commodité, cette dernière m'a livré un texte en réponse à mes questions. Les citations que j'en tire participent du récit et apportent le double point de vue, de l'architecte et de l'agricultrice, à la description paysagère. Enfin, parce que ma pratique de la marche s'assortit de celle du dessin, qui est un acte individuel et plutôt solitaire, je me dois de préciser que les deux formes de rencontres (paysagère d'un côté 
et des agriculteurs de l'autre) se sont organisées en deux temps distincts mais reliés entre eux par des documents (cartes et dessins), supports d'entretiens.

Saisir, décrire et représenter les paysages de cette exploitation agricole métropolitaine est le sens que j'ai voulu donner à ce voyage et à cette contribution en rassemblant un peu de matières premières à ce qui pourrait potentiellement devenir un travail plus large de recherche et de projet.

\section{L'arrivée au Reyerhof, premiers contacts avec les paysages}

\section{À deux pas du centre-ville de Stuttgart}

Depuis Schlossplatz - une des places du centre-ville de Stuttgart - la ligne $5 \mathrm{du}$ Stadtbahn ${ }^{8}$ ne met pas plus de quinze minutes pour gagner Rohrer Weg l'arrêt le plus proche de la ferme. Quittant le fond du thalweg occupé par le centre-ville de Stuttgart (Stuttgart-Mitte), celle-ci gravit la pente escarpée du coteau avant d'atteindre le plateau où se trouve Möhringen. La commune est en effet installée en tête du bassinversant du Körsch, à l'endroit où le plateau qui domine la rive gauche de la vallée du Neckar se creuse et où différents cours d'eau confluent pour donner naissance à la vallée du Körsch (Körschtal). À une altitude voisinant les $400 \mathrm{~m}$, le terroir, faiblement vallonné, est décrit par André Birre en 1969 comme "un sol de limon de $8 \mathrm{~m}$ d'épaisseur, frais et fertile, convenant bien pour la culture des choux à choucroute qui font la renommée du pays ». La culture des choux est aujourd'hui toujours bien présente et, à la belle saison, on peut en apprécier l'étendue bleutée aux abords de Möhringen.

7 À l'approche de l'arrêt Rohrer Weg, le Stadtbahn ralentit sa progression. C'est à ce moment-là que je les aperçois, depuis les vitres du wagon. D'abord une ribambelle de vélos en bout de champ, des caisses en plastique déposées dans l'herbe derrière le fourgon estampillé du "Landwirtschaft in der Stadt für die Stadt», puis elles : cinq à six personnes, les pieds dans la paille, courbées, accroupies, à cheval dans les fraisiers. J'apprendrai par la suite que c'est jour de récolte. Salariés, gestionnaires et apprentis de la ferme s'activent à déposer les précieux fruits rouges dans des barquettes en carton recyclé. En cette veille de week-end, il s'agit de faire provision de fraises pour fournir le magasin de la ferme, tandis que le reste de l'abondante fructification sera transformé en coulis pour parfumer glaces et yaourts de la ferme.

\section{«Arrêt Rohrer Weg "}


Figure 1. Rohrer Weg, un espace agricole en vue, traversé par le Stadtbahn en lisière de StuttgartMöhringen

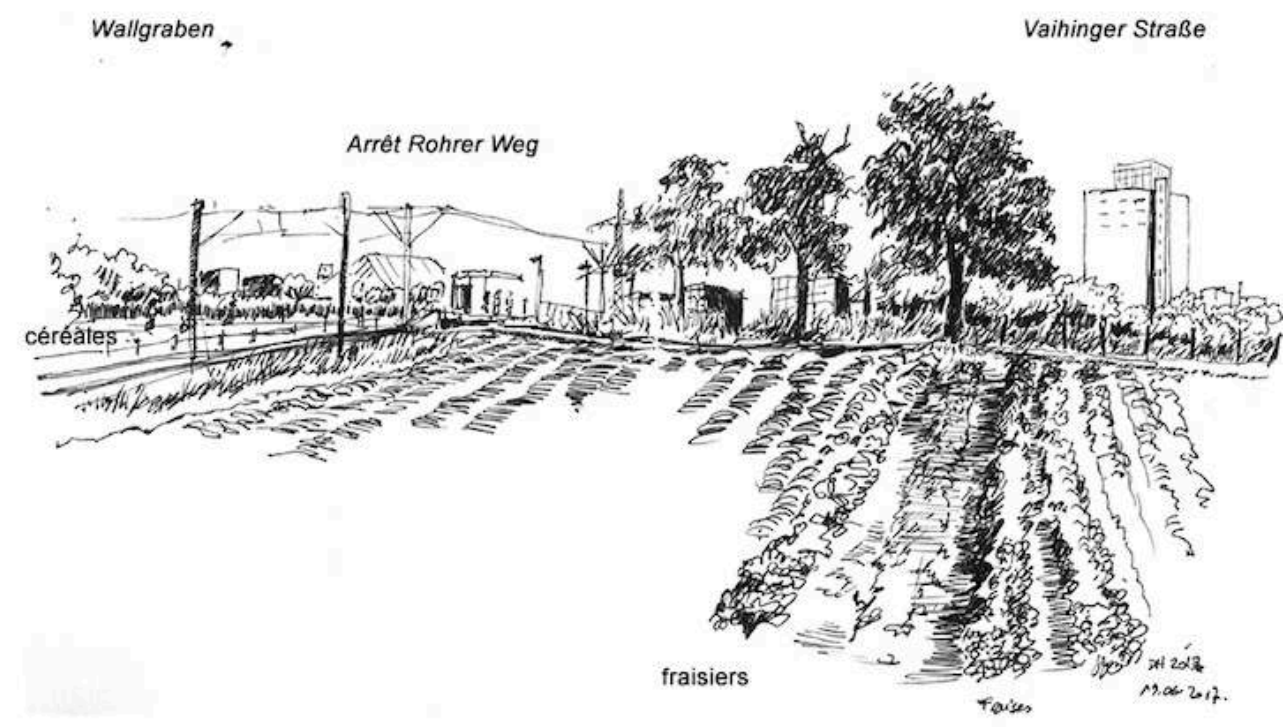

Source : Dominique Henry, 2017.

8 La particularité de la station, où je descends, est d'être littéralement aux champs. Il s'agit, ni plus ni moins, d'un quai au milieu d'un espace agricole suffisamment étendu pour offrir des vues larges paysagères et un recul sur les différents quartiers ouest de Möhringen. L'observation à la ronde donne à voir, à l'est, un horizon encadré par les toitures du centre-bourg de Möhringen (Möhringen-Orstemitte), et dominé par la skyline des tours de logements de Fasanenhof, tandis qu'émergent des frondaisons, côté ouest, les bâtiments de la zone d'activités tertiaires de Wallgraben.

En passe d'arriver au Reyerhof, me voici immergé dans les paysages que cette ferme participe à fabriquer au sud de Stuttgart; ils se caractérisent par un espace agricole où alternent cultures sarclées, céréales et herbages dans les vergers de vieux pommiers. Ces paysages, résultat du contact rapproché de réalités habituellement si différentes une diversité de cultures, des travailleurs au champ sur fond de scène urbain -, m'étonnent, m'enchantent. Ce type de foisonnement et de rapport d'échelle lié à une agriculture "paysanne " et maraîchère existe peu dans et aux abords des villes françaises comme Bordeaux, Strasbourg, Lille, ou Niort par exemple, davantage dominés par une agriculture de plus grandes surfaces. Le fait n'est pas si commun pour retenir mon attention et motiver mon voyage dans les terres cultivées par la ferme, dont la distribution spatiale m'engage à effectuer un tour de ville de Möhringen.

Cette idée du tour de ville n'est d'ailleurs pas pour me déplaire. En effet, elle se présente à moi comme une adaptation à la pratique du "tour de pays ${ }^{9}$ ", chère à l'agriculteur, à l'agronome (Deffontaines, 1998) et à certains paysagistes (Ambroise et al., 2000 ; Henry, 2004), pour s'informer de l'état des cultures ou du paysage, et décider des pratiques ou des projets à mettre en œuvre. 


\section{Tour de ville à Möhringen}

Figure 2. Un voyage en terres urbaines cultivées par le Reyerhof, autour de Möhringen

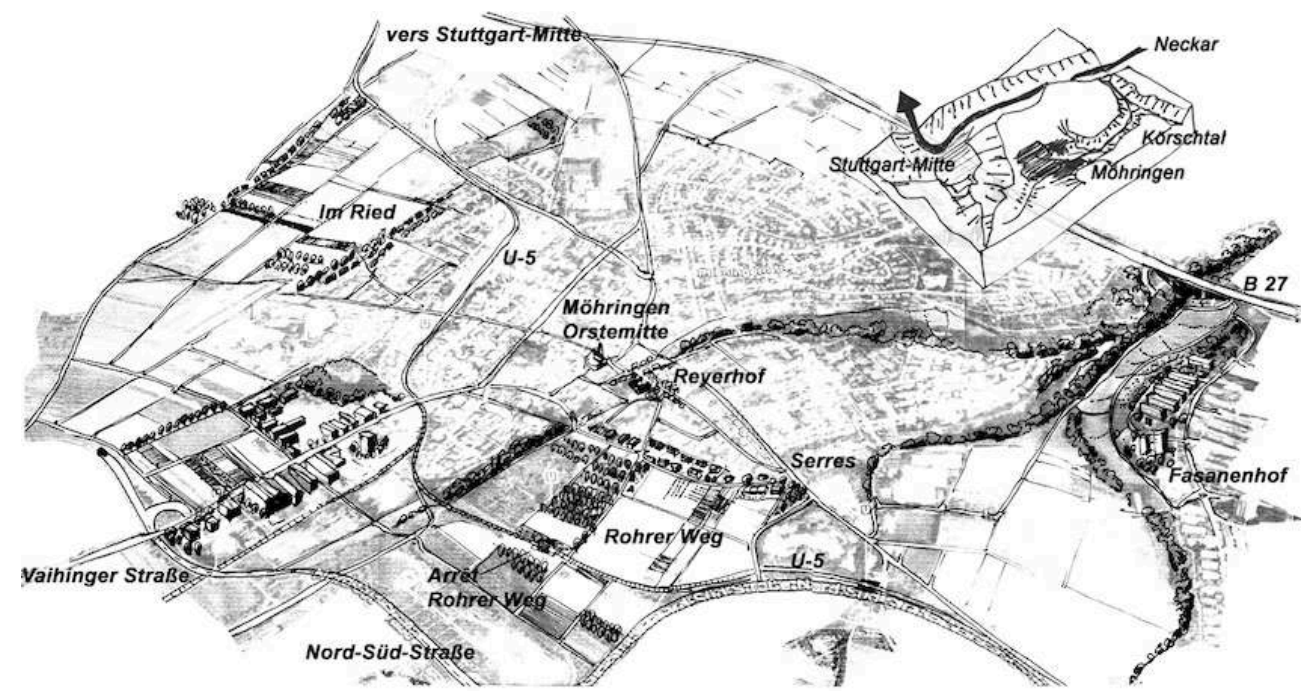

Source : Dominique Henry, 2017.

11 En prenant de la hauteur, on comprend l'imbrication des champs et du bâti qui conditionne cette agriculture dans la ville. L'espace agricole y apparait circonscrit, enserré au cœur d'un ensemble urbain au dessin complexe fait de divers infrastructures de transport, types et densités de constructions. Au nord, au sud comme à l'ouest, les terres agricoles sont en contact avec des fronts bâtis bien différents en hauteur, implantation, fonction et ambiance. C'est la volonté de saisir les contrastes du paysage urbain lui-même, dans ses relations quasi immédiates et frontales avec l'organisation des cultures, elles-mêmes également différenciées, qui a décidé du tour qu'allait prendre mon voyage. Il s'organise par la visite successive de quatre quartiers - Roher Weg, Vaihinger Straße, Im Ried, Fasanenhof - avant de se terminer dans la cour de la ferme.

\section{Rohrer Weg}

12

Outre le fait d'être traversé par le Stadtbahn, ce quartier a la particularité d'être longé, côté sud et ouest, par la Nord-Süd-Straße (grande route connectée à l'autoroute A8), et d'être délimité, côté est et nord, par un tissu d'habitat résidentiel composé de maisons individuelles et de petits immeubles collectifs. Quadrillé de chemins agricoles (ouverts au public), c'est non seulement un espace agricole très en vue, mais aussi très fréquenté, tant pour les loisirs qu'à travers la mobilité quotidienne. Le morcellement parcellaire y est important. Il participe de la juxtaposition des cultures (céréales, fraisiers, pommes de terre, fleurs à couper et légumes), qui avec les structures linéaires des vergers de vieux pommiers composent un paysage particulièrement attractif. Alina témoigne en ce sens : "Quand je travaille dans cette lisière de ville et de campagne, je suis à chaque fois fascinée par cette organisation spatiale. Je me demande ce que pensent les gens, dans le Stadtbahn, quand ils nous voient là-bas dans les cultures. Arrivent-ils à voir ce que nous récoltons ? Sont-ils capables d'établir une relation entre notre travail et leur nourriture? Est-ce qu'ils nous envient notre travail ou sont-ils au 
contraire heureux de ne pas devoir le faire? Ce qui me plaît dans cette parcelle, c'est que les gens passent par là et peuvent voir cette agriculture "animée" par les gens qui travaillent la terre.»

Figure 3. Rohrer Weg, la diversité paysagère des parcelles maraîchères du Reyerhof au contact du quartier résidentiel

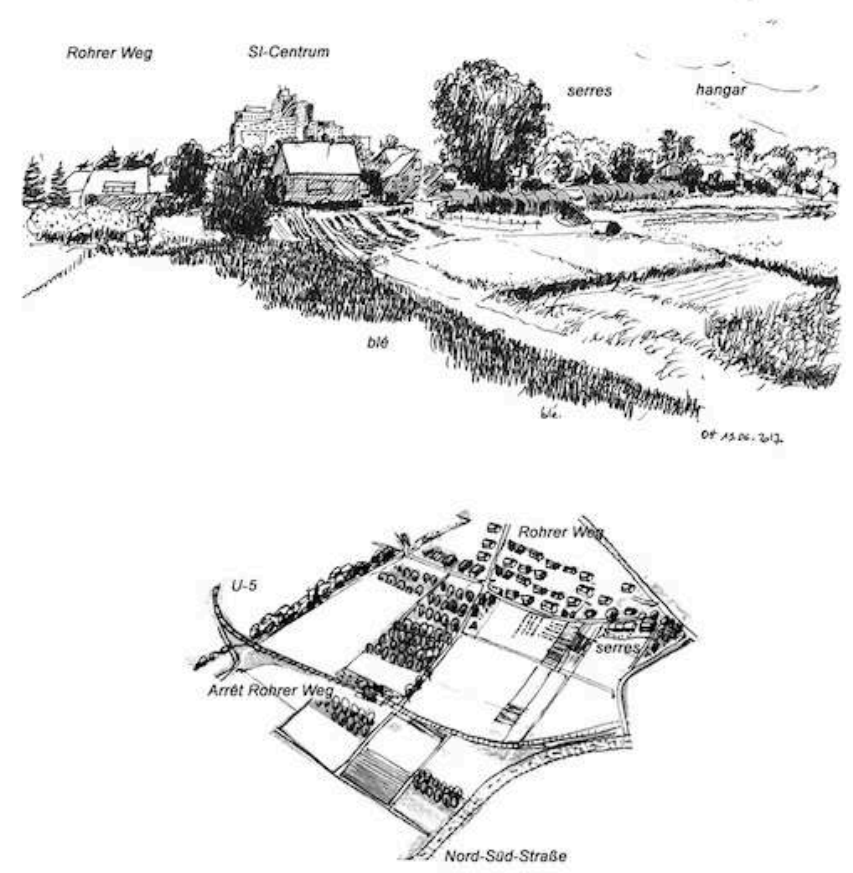

Source : Dominique Henry, 2017.

13 Le Reyerhof participe de cette polyculture en lisière des quartiers résidentiels, en cultivant, dans le prolongement des $600 \mathrm{~m}^{2}$ de serres froides (tunnels bâchés), les légumes (Feingemüse) requérant le plus de soins quotidiens (arrosage, récolte ajustée, etc.) tels que salades, tomates courgettes, etc. Cet ensemble de champs est celui qui est en lien fonctionnel le plus direct avec les bâtiments du siège d'exploitation : il est le plus proche, dispose d'un point d'eau pour l'arrosage et est structuré par un hangar de stockage des machines (Maschinenhalle).

\section{Vaihinger Straße}


Figure 4. Vaihinger Straße. Saisissants contrastes des étendues de choux et de céréales traversées de chemins cyclables, avec cette lisière de ville

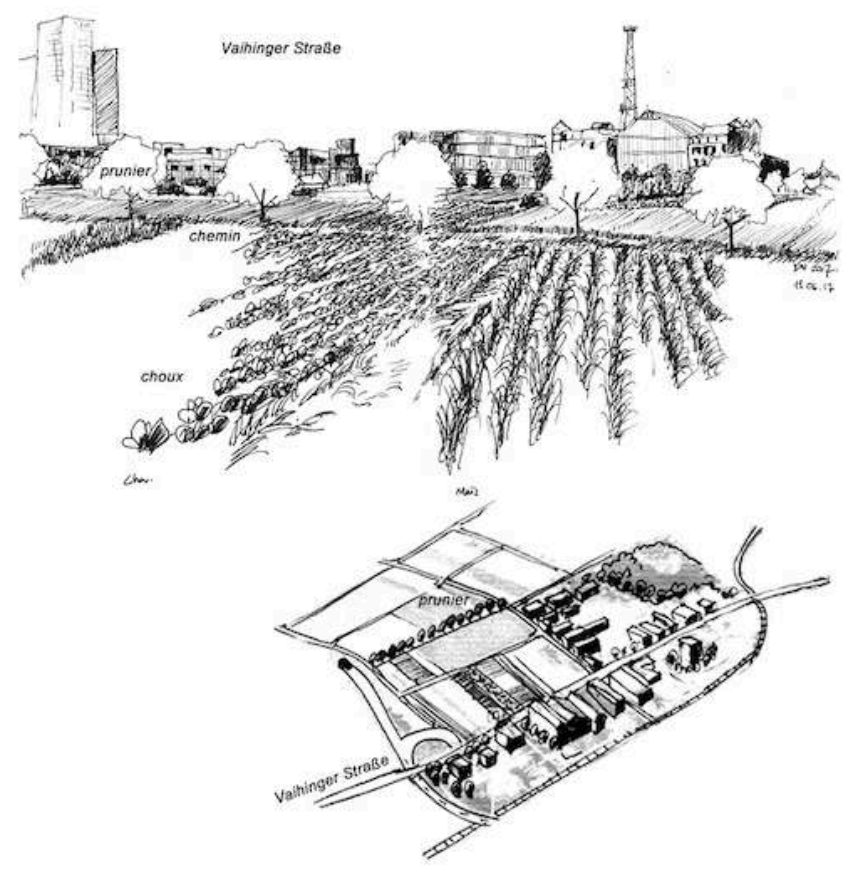

Source : Dominique Henry, 2017.

À peu de distance, le quartier agricole de Vaihinger Straße offre un tout autre paysage. Cela autant pour les cultures qui s'organisent ici en plus grandes parcelles géométriques, que pour le front urbain qui le domine. Au sud, le long de l'avenue, s'organise un ensemble de bâtiments d'activités tertiaires, de 6 à 10 étages, d'où émerge l'étonnante architecture de Gottfried Böhm ${ }^{10}$. Côté est, le front urbain de ce quartier agricole est marqué quant à lui par une récente opération d'habitats collectifs, dont le plan en peigne ouvre de longues perspectives sur le paysage agricole. Seul un chemin de desserte en enrobé opère la transition entre les cultures et les pieds d'immeubles. Il est très fréquenté certes par les engins agricoles, mais surtout par les piétons et les cyclistes, qui forment, à certaines « heures de pointe », un trafic non négligeable.

Entre l'alignement de pruniers et Vaihinger Straße, le Reyerhof y cultive ici, selon les rotations, choux, blé, carottes, pommes de terre et oignons. Interrogés sur ce que représente pour eux une telle proximité urbaine, les agriculteurs reconnaissent des côtés positifs comme des désagréments. Traverser des routes fréquentées avec des engins agricoles n'est pas toujours facile. De même, il n'est parfois pas aisé de concilier exigence agricole et vie urbaine, quand la préférence est par exemple donnée à une récolte en sol sec. Alina se souvient encore du nuage de limon soulevé par la trieuse de pommes de terre à l'automne 2016 et rejeté par le vent sur les véhicules empruntant Vaihinger Straße. Lukas témoigne aussi de récoltes sauvages parfois pratiquées sur quelques rangs. Mais la proximité urbaine peut aussi avoir des effets positifs, notamment sur la population qui peut s'étonner de ce que représente le travail agricole quand il est en vue, sur le devant de la scène pourrais-je aussi dire. Une employée relate cette anecdote d'un chantier de récolte de carottes organisé un jour férié pour parer 
aux pluies annoncées : «Beaucoup de promeneurs s'arrêtent pour observer le travail de la machine qui sort les carottes, pour regarder le tracteur avec la remorque et ce grand groupe que nous formons dans le champ : employés, stagiaires, membres de la SoLaWi. Un couple âgé s'arrête et s'étonne de "tous ces jeunes gens" qui participent au chantier.»

\section{Im Ried}

Figure 5. Im Ried, la diversité des cultures du Reyerhof associée au morcellement des parcelles crée un paysage à échelle humaine en lisière de Möhringen, petite ville de 31000 habitants

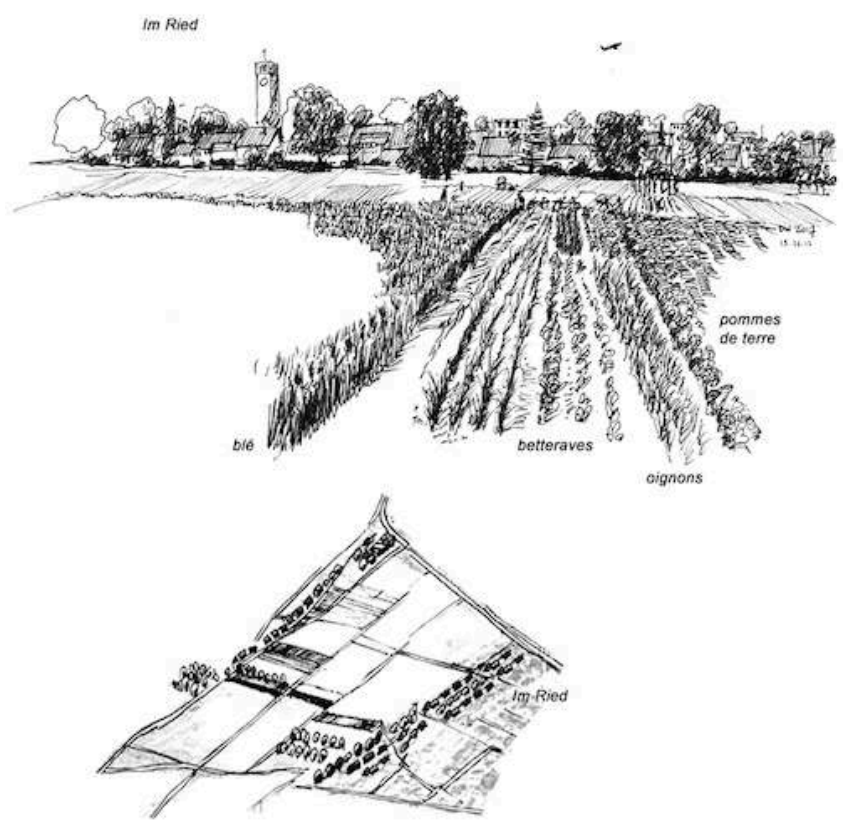

Source : Dominique Henry, 2017.

Les terres cultivées au nord de la commune sont celles qui expriment le mieux l'idée de plateau agricole. Sans relief perceptible, le paysage est ici animé par la silhouette villageoise de quartiers d'habitats pavillonnaires, au nord comme au sud, où abondent des jardins et leur végétation variée. Cette dernière fait en partie écho aux pépinières d'arbres et d'arbustes de pleine terre qui parsèment l'ensemble de champs entrecoupés de quelques alignements relictuels de pommiers. Au sein des surfaces emblavées, les parcelles de la ferme se remarquent par les rangs d'oignons, de betteraves, de carottes et de pommes de terre qui apportent rythmes et diversité. Les agriculteurs du Reyerhof ont conscience de ce qu'implique, en termes de paysage, ce mode de culture biodynamique, basé sur l'interaction et la diversité interspécifique. «Selon la rotation des cultures sur huit ans, chaque année, une culture différente est cultivée sur cette même parcelle. Cela garantit non seulement un sol sain et moins de parasites, mais aussi chaque année une nouvelle apparence dans la structure du paysage ", souligne Lukas. Faisant part de son expérience paysagère, Alina témoigne en ce sens : "Je me rends compte, en particulier lors de voyages ferroviaires à travers le pays, combien les 
agriculteurs façonnent les paysages. Les déserts agricoles, ailleurs, me font prendre conscience de ce qu'apporte et fabrique le Reyerhof en tant que ferme en polycultureélevage produisant des cultures diversifiées sur des petites parcelles. »

\section{Fasanenhof}

Figure 6. Fasanenhof, un vallon herbager où la ferme fauche les prés entre quartier d'affaires (à gauche) et grand ensemble d'habitat (à droite)

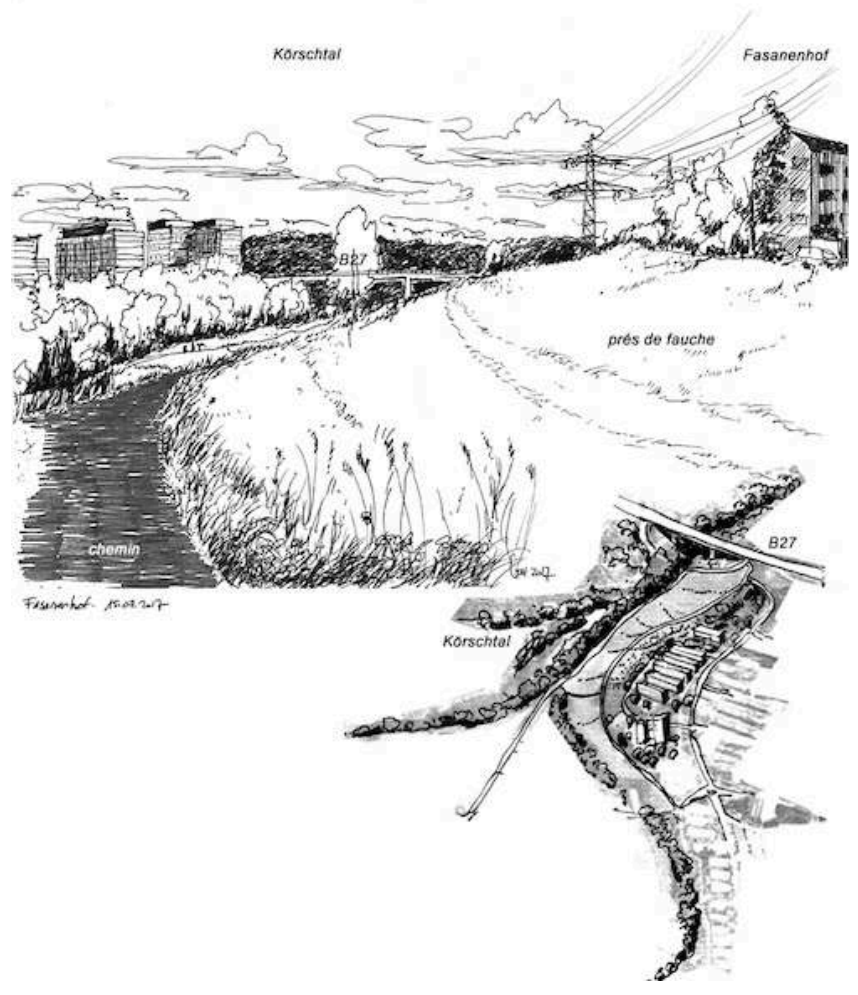

Source : Dominique Henry, 2017.

$17 \mathrm{Au}$ sud, le quartier de Fasanenhof, offre à mon tour de ville un tout autre paysage. Il est marqué par le relief plus accentué de la vallée du Körsch, barré du pont routier de la B27 (une pénétrante donnant accès direct au centre de Stuttgart) et hérissé, à l'horizon, de tours. Ce sont, sur la rive gauche, des bâtiments de bureaux, tandis qu'en rive droite, Fasanenhof correspond à un grand ensemble, composé de barres et de tours de logements, organisé selon les principes de l'urbanisme moderne. À son pied, en basversant et fond de vallon, s'étirent des prés de fauche en partie exploités par le Reyerhof. Le foin qui y est récolté participe à constituer la réserve de fourrage nécessaire à l'alimentation hivernale des vaches laitières de la ferme.

Il est étonnant de constater ici combien la présence des terrains agricoles est intégrée à la composition d'ensemble du quartier, dont les façades s'ouvrent largement sur le vallon. De fortes continuités d'espaces et de circulations ont été pensées et aménagées de sorte que les allées et pelouses des pieds d'immeubles rejoignent chemins et herbages de l'espace agricole. 


\section{Côté ferme, une cour animée}

Figure 7. Au cœur de l'ancien noyau villageois de Möhringen, la cour du Reyerhof est insolite et animée

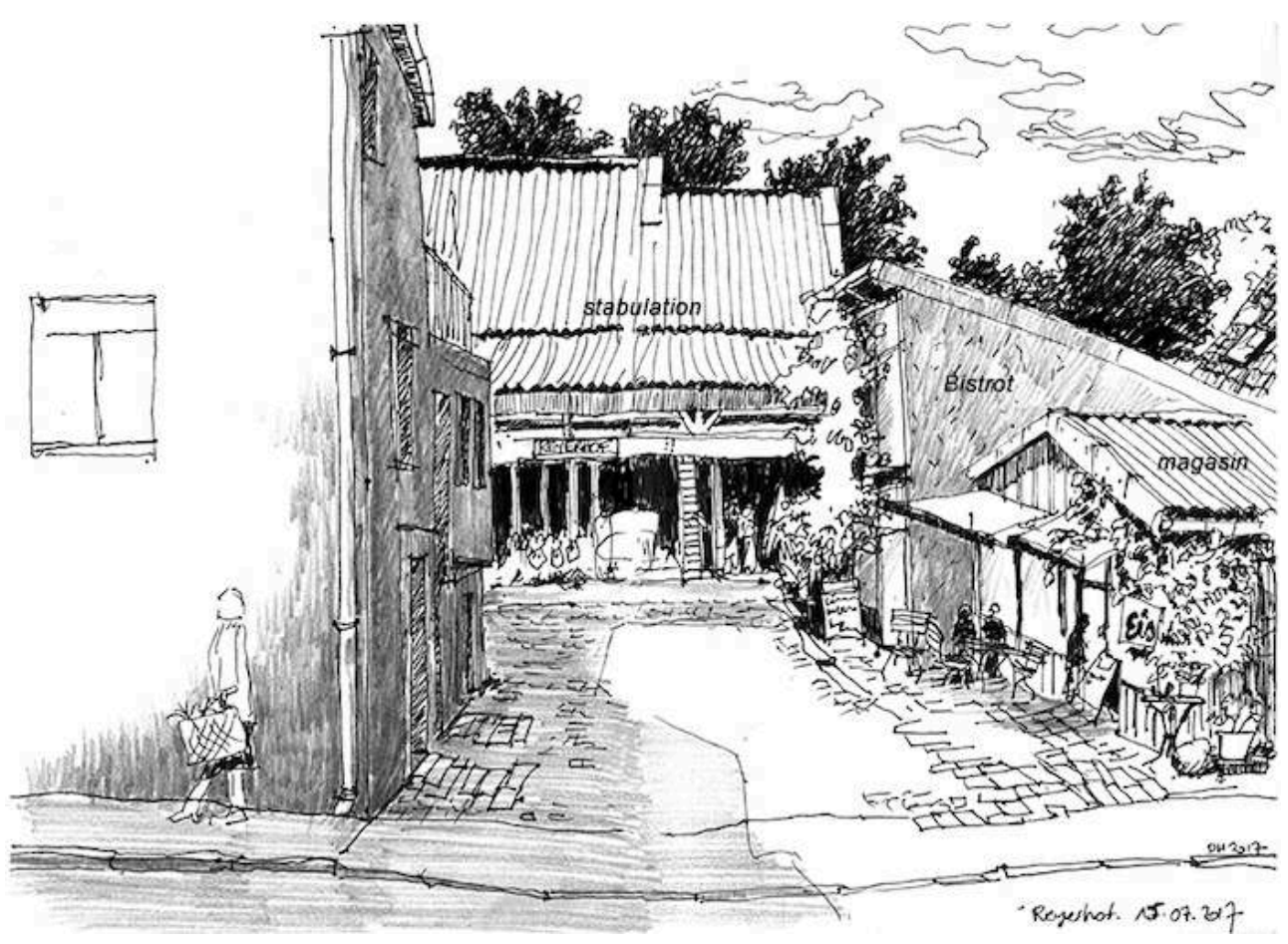

Source : Dominique Henry, 2017.

Dans cette rue de village d'où pointe le clocher et où les maisons basses se mêlent à de petits immeubles d'habitation de deux à trois étages, rien n'annonce particulièrement les bâtiments de la ferme, avant que le dégagement de la cour en forme de U ne s'ouvre perpendiculairement à la voie. Et là : surprise! C'est l'agitation d'une petite place de village. Des clients entrent et sortent du Hofladen ${ }^{11}$, repartent ou rejoignent d'autres personnes installées à la terrasse du Bistrot ${ }^{12}$, qui commercialisent tous deux les produits de la ferme, le tout devant une dizaine de paires d'yeux qui, de la stabulation au fond de la cour, ruminent tranquillement. Suivant les heures de la journée, il faut ajouter à cela le passage du tracteur et du fourgon ainsi que les allées et venues des employés et apprentis qui, entre deux départs aux champs à vélo, prennent leur repas et pour certains habitent - dans l'aile gauche de cet ensemble architectural agricole complexe. Celui-ci abrite en outre bureau, cuisine, salle à manger ${ }^{13}$ et, en sous-sol, la salle de traite et les locaux-laboratoires de la transformation laitière.

Il est de moins en moins courant de voir des bâtiments agricoles en fonctionnement imbriqués dans un ancien noyau villageois. Cela représente pour cette ferme un avantage commercial indéniable. Les vaches constituent une attraction appréciée de toute la population enfantine (mais pas que) des environs. Leur rendre visite est aussi souvent l'occasion de déguster une glace fabriquée avec leur lait. Mais cela demande aussi à l'agriculteur d'adapter ses pratiques (horaires, propreté de la chaussée) au voisinage urbain, et de développer une certaine dextérité pour manœuvrer tracteur et remorque dans une étroite rue encombrée de véhicules en stationnement. 


\section{Diversités}

21 Je reviens surpris de ce tour de ville par tant de diversité. Elle est celle des paysages agricoles et urbains qui sont bien différents d'un quartier à l'autre. Elle est celle aussi des cultures et des modes de commercialisation au sein de cette ferme, qui semblent avoir tiré parti de sa situation urbaine. Le Reyerhof rassemble en effet des productions maraîchère $(4 \mathrm{ha})$, céréalière et de champs $(15 \mathrm{ha})$ et fourragère $(17$ ha dont 8 ha de vergers) pour un total de $36 \mathrm{ha}$, associées à un élevage de 10 vaches laitières, mobilisant le travail de 7 personnes. La production est entièrement vendue en direct (après transformation d'une partie du lait en fromage frais, yaourts et glaces) au magasin de la ferme et au Bistrot qui comptent 10 employés (5 équivalents temps plein) et à travers la fourniture hebdomadaire de 280 paniers à la SoLaWi de Stuttgart. La commercialisation des produits mise donc sur l'important vivier de consommateurs potentiels proches ${ }^{14}$ ainsi que sur une démarche de qualité qui se retrouve à de multiples niveaux : qualité de la production alimentaire qui répond au cahier des charges de l'agriculture biodynamique (label Demeter) depuis $1957^{15}$, qualité et soin apportés à l'architecture agricole et à ses abords, et enfin qualité et diversités des paysages au voisinage étroit des quartiers d'affaires et d'habitations. Le dispositif est riche, complexe. Il est celui d'une exploitation agricole devenue de plus en plus urbaine de part son interaction avec les espaces de la ville et avec les citadins et plus particulièrement les adhérents de la SoLaWi.

L'investigation demanderait bien évidemment à être poursuivie pour apporter des réponses aux questions que fait naître ce récit. Comment, par exemple, cette promiscuité agri-urbaine influe (ou non) sur les pratiques des agriculteurs du Reyerhof et sur les modes de culture? Qu'est-ce que cela implique en matière d'organisation du travail agricole? Cette visibilité paysagère des terres cultivées, à l'égal d'un espace public inséré dans le tissu urbain, influence-t-elle l'agriculteur dans ses choix techniques, dans sa conscience paysagère? En retour, les membres de la SoLaWi ont-ils conscience de cette étonnante imbrication du Reyerhof avec Suttgart-Möhringen? Quelles sont leurs motivations dans le soutien à cette agriculture et à cette ferme?

Il s'agirait, en outre, d'élargir la réflexion et de tenter la comparaison avec d'autres exploitations agricoles et d'autres situations métropolitaines.

\section{Métropole cherche rapprochements agricoles - et inversement si affinités}

24 Au terme de ce voyage en terres urbaines cultivées, il me semble nécessaire de formuler quelques réflexions à titre de conclusion provisoire.

\section{Quand la ville se rapproche... mais de quelle dynamique urbaine parlons-nous?}

Est-il nécessaire de revenir sur le glissement opéré par la ferme et son espace de production, d'un fonctionnement familial en lien avec les terres au pourtour d'un village, Möhringen, vers une agriculture enserrée dans l'espace urbain de StuttgartMöhringen, conduite par une petite entreprise agricole qui produit, transforme et vend 
une alimentation en direct et pour partie en mode solidaire. Cette agriculture est ainsi devenue urbaine par rapprochement de la ville. Mais il y a différentes manières de voir celui-ci. Il y a le rapprochement spatial, subi quand l'urbanisation empiète, soustrait des terrains; et il y a le rapprochement social qui, lui, ouvre de nouveaux horizons de collaborations et d'échanges.

La première dynamique urbaine, est celle, connue, qui phagocyte les terrains, ou qui poursuit d'autres projets, parfois environnementaux, sans que l'agriculture soit pour autant une préoccupation. Ce qui s'est produit avec les terres du Rohrer Weg est en cela éclairant. L'histoire débute en mars 2002 lorsque des élus (de droite) de Möhringen demandent au conseil municipal de modifier le plan d'urbanisme pour accroître la partie constructible au Rohrer Weg. Le 26 juin, le conseil municipal valide le projet de construction contre les voix des élus écologistes et de gauche. Le lendemain, 27 juin, est créée sans attendre l'association Sauvez les champs et les vergers au Rohrer Weg ${ }^{16}$. Elle sera à l'origine d'une pétition, d'un rassemblement citoyen, et de la visite des vergers par quelques grands hommes politiques. Après diverses péripéties politiques, toute la surface est sortie des enjeux de l'urbanisation en août 2005 par son classement en zone naturelle. L'argument principal avancé est celui... de la protection des oiseaux et du climat $^{17}$. La sauvegarde du potentiel productif des champs et des vergers ne semble pas avoir été la motivation première, même si, d'une certaine manière, la revendication et la réglementation écologique auront permis le maintien de l'espace agricole.

La seconde dynamique est celle qui a conduit un groupe de citadins à se rapprocher de la ferme. Et non l'inverse. C'est en effet d'abord des consommateurs engagés et porteurs d'une éthique pour leur alimentation qui se sont mis en recherche d'une exploitation agricole "en ville", prête à s'engager avec eux dans une démarche solidaire. En effet, pour Alina, qui a participé à l'aventure depuis son début, «la production devait être transparente pour nous et de proximité par conviction écologique. De plus, il y avait un fort désir d'avoir un contact personnel avec la ferme et de pouvoir participer au travail. Un échange continu entre le producteur et les consommateurs devait permettre le développement conjoint du projet. Donc, notre objectif était de trouver une ferme bio proche de la ville. » Et de poursuivre sur ce qui a conduit les consommateurs au Reyerhof: "Il y avait trois fermes potentielles, dont l'une fut en fait rapidement éliminée en raison de la distance $(30 \mathrm{~km})$. Les deux autres étaient de la même manière proche de la ville et c'est le Reyerhof qui, la première, s'est finalement aventurée dans l'expérience avec nous. Le fait que la ferme soit en ville et accessible par les transports publics a rendu beaucoup plus simples les rencontres pour faire connaissance et pour élaborer ensemble le projet. » 


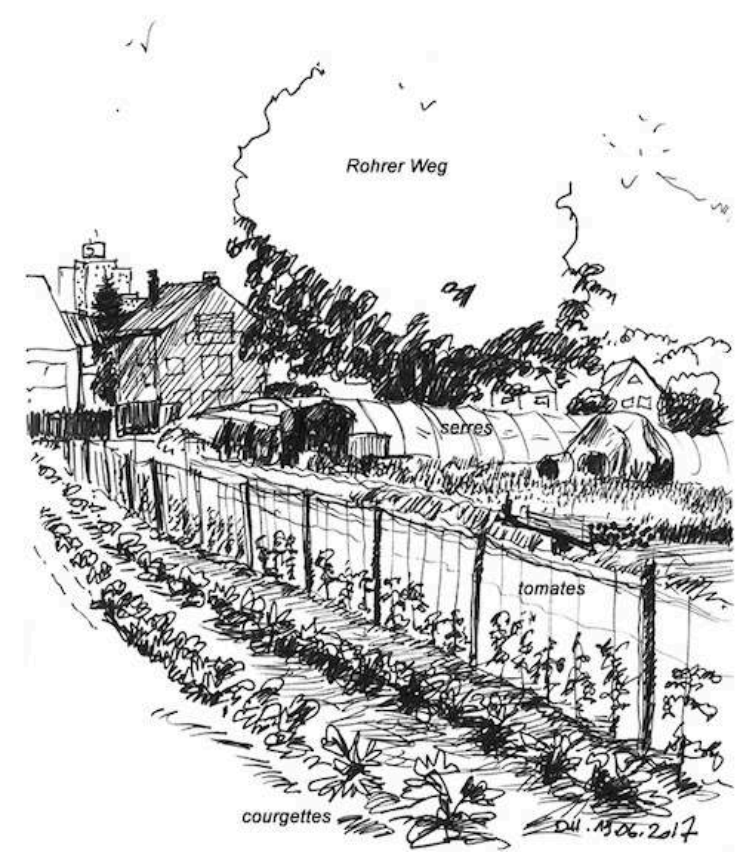

Source : Dominique Henry, 2017. engagement réciproque. Il s'est traduit au sein de la ferme par un ajustement de ses productions maraîchères par exemple. En effet, pour composer les paniers hebdomadaires, la ferme s'est rapprochée des désirs exprimés par les consommateurs, en diversifiant par exemple la gamme des légumes, et en garantissant encore plus l'origine des produits : c'est par exemple le cas pour la farine, estampillée Reyerhof, exclusivement issue du blé produit par la ferme, avec laquelle un boulanger local produit une fournée spécifique de Hofbrote (pains de la ferme) qui garnissent le magasin et les paniers tous les jeudis. Dans cette même idée, Lukas étudie par ailleurs la possibilité de produire du seigle pour compléter l'offre en pain.

L'économie solidaire qui est à la base de la SoLaWi garantit à l'exploitant agricole de vendre sa production, qu'elle soit prolifique ou moins, à un prix équitable, décidé à l'avance lors d'une réunion annuelle des adhérents durant laquelle est voté le budget. À deux reprises, les membres de la SoLaWi, ayant à cette occasion accès aux comptes, ont exprimé le souhait de voir augmenter le tarif horaire des salariés de la ferme. Par vote, le groupe a décidé d'en supporter le coût à travers l'augmentation du prix du panier.

$\mathrm{Au}$ final, la proximité géographique avec la ville qui, à certains égards, est contraignante peut être aussi un avantage. À condition toutefois de savoir en tirer profit.

\section{Un projet agricole biodynamique}

Revenons enfin sur un dernier point, crucial en agriculture, et plus encore pour celle qui est urbaine, celui du foncier. "L'espace est une denrée rare et un grand nombre 
d'acteurs est intéressé par les terres, construites ou non construites et, par conséquent, le foncier est cher ", comme le souligne Alina.

La réponse adoptée par la ferme en matière de foncier mérite d'être observée avec attention. Son assise foncière est de 36 ha qui, en dehors d'un seul hectare, correspondent à des locations. Elle était de 20 ha en 1969 et de 29 ha en 1998. L'augmentation est constante mais mesurée. Elle reste en deçà de la superficie moyenne, toutes exploitations confondues, qui est de l'ordre de $61 \mathrm{ha}^{18}$ en France comme en Allemagne. Elle est aussi bien loin de l'agrandissement à outrance promu par certains systèmes de production qui y recherchent la rentabilité économique. La logique adoptée ici est celle de l'optimisation des terrains disponibles: la faible augmentation des surfaces cultivées y est modulée par une forte augmentation de la valeur ajoutée sur la production agricole. Mais cette logique n'est pas que foncière. Elle renvoie, en définitive, à la capacité du projet agricole d'être - et de rester - vivant et dynamique - une autre manière peut-être de définir une agriculture biodynamique c'est-à-dire en mesure de s'adapter, entre contraintes et avantages, et de tirer profit, à tous niveaux, de son insertion urbaine.

Je tiens à adresser mes chaleureux remerciements à Alina, dont le texte écrit m'a été d'une grande utilité, à Lukas pour ses réponses concrètes et pratiques, à Katrin pour sa précieuse aide à la traduction, ainsi qu'à tous les êtres vivants du Reyerhof qui m'offrent, régulièrement, de quoi me régaler.

\section{BIBLIOGRAPHIE}

Le projet agricole biodynamique du Reyerhof, visible dans les paysages agricoles et urbains de Möhringen, ouvre sans doute quelques sillons fertiles.

Ambroise, R., Bonneaud, F., Brunet-Vinck, V., Agriculteurs et Paysages. Dix exemples de projets de paysage en agriculture, Dijon, Educagri, 2000, $207 \mathrm{p}$.

Augé, M, La Vie en double, Paris, Payot et Rivages, 2011, 268 p.

Donadieu, P., Campagnes urbaines, Arles, Actes Sud, 1998, 224 p.

Bernetti, I., Fanfani, D., Monacci, F., Poli, D., Rubino, A., « Le parc agricole comme instrument de l'aménagement stratégique multifonctionnel des espaces périurbains de la Toscane centrale », colloque international « Les agricultures périurbaines, un enjeu pour la ville. Vers des projets de territoire », Nanterre, 10-12 octobre 2007.

Birre, A, « Chez M. Karl Reyer. Petite ferme allemande de Möhringen », dans Une politique de la terre, Marcq-Lille, Éditions Vie et Action, 1969, p. 207-209.

Boutleux, D., « Les champs urbains. Réinventer les mythologies urbaines », Openfield, $\mathrm{n}^{\circ} 2$, juin 2013, URL : https://www.revue-openfield.net/2013/06/23/les-champs-urbains-reinventerles-mythologies-urbaines/.

Deffontaines, J.-P., Les Sentiers d'un géoagronome, Paris, Arguments, 1998, 360 p 
Étienne, M, « Voyage à Kraftwerk », Criticat, n 11, 2013, p. 13-23

Henry, D., « Un voyage paysagiste en agriculture », Revue d'Auvergne, vol. « Des paysages pour le développement local. Expériences et recherches innovantes dans le Massif central », n 571, 2004, p. 115-131.

Janin, R., La Ville agricole, Pantin, Éditions Openfield, 2017, 70 p.

Janin, R., « Trois enclaves agricoles dans la périphérie de Lyon, vers des agricultures urbaines ? ", Projets de paysage, $\mathrm{n}^{\circ} 2$, juin 2009, en ligne : http://www.projetsdepaysage.fr/

trois_enclaves_agricoles_dans_la_p_riph_rie_de_lyon_vers_des_agricultures_urbaines_.

Laverne, T., «L'expérience du Triangle vert, projet de territoire agri-urbain durable », Cahier

thématique. Agriculture métropolitaine/Métropole agricole, 2011, n 11, ENSAP de Lille, p. 257-271.

Masboungi, A. (dir.), Extension du domaine de l'urbanisme. Frédéric Bonnet, Grand Prix de l'urbanisme 2014, Paris, Parenthèses, 2014, 144 p.

\section{NOTES}

1. http://www.trianglevert.org/

2. Voir l'article de Monique Toublanc et Monique Poulot « Les territoires agriurbains en Île-deFrance: entre paysage ordinaire, paysage agricole et paysage alimentaire? publié dans ce numéro 17 de Projets de paysage, URL : https://journals.openedition.org/paysage/4782

3. https://www.reyerhof.de/

4. Littéralement : " Landwirtschaft in der Stadt für die Stadt ».

5. Je voudrais ici signaler ma dette envers l'architecte et illustrateur Martin Étienne, dont le "Voyage à Kraftwerk ", une visite dessinée de la coopérative d'habitat, publiée dans la revue Criticat en 2013, aura inspiré mon voyage autour du Reyerhof - ici à la belle saison! : http:// jhapa.eu/wp-content/uploads/2016/09/criticat11_Martin-Etienne.pdf.

6. Au sens exprimé par Marc Augé (2011) : «"Faire” une rencontre, ce pourrait vouloir dire, au contraire, la fabriquer, la façonner, faire quelque chose avec ce qui n'était rien qu'un pur hasard. Je croise mille personnes chaque jour, je ne les rencontre pas. Si je les rencontre, c'est que quelque chose en eux m'a retenu, qu'ils me "disaient quelque chose" et que, en somme, c'est moi qui ai voulu faire quelque chose de rien, faire du hasard un événement et reconnaître un inconnu.»

7. Pour "Solidarische Landwirtschaft", équivalent de l'Amap en France. Pour plus d'info: https://www.solidarische-landwirtschaft.org/de/startseite/

8. Métro aérien et souterrain reconnaissable à sa couleur jaune et dont le réseau s'inscrit à l'échelle de l'agglomération.

9. On trouve aussi le nom de «tour de plaine ».

10. Architecte allemand contemporain, né en 1920, qui reçut de nombreux prix, dont le prestigieux Pritzker Prize en 1986.

11. Magasin bio, à la ferme.

12. Nom commercial du restaurant transformant une partie des productions de la ferme.

13. Les repas (petits déjeuners et déjeuners) y sont pris en commun.

14. Signalons que la commune de Möhringen, qui appartient au 23 quartiers (Stadtbezirke) de Stuttgart, compte 31000 habitants sur les 610000 de l'agglomération.

15. On peut lire à ce sujet l'intéressant témoignage d'André Birre, venu en 1969 chez Karl Reyer, visiter une «Petite ferme allemande à Möhringen»: «M. Reyer a commencé la culture 
biodynamique il y a 12 ans, à la suite d'une conférence à laquelle il avait assisté et parce qu'il avait ressenti chez lui les inconvénients de la fertilisation chimique. »

16. Littéralement : « Rettet die Felder und Streuobstwiesen am Rohrer Weg ». Plus d'information, se reporter à l'historique complet retracé par l'association sur son site : http://rohrer-weg.de/ Chronologie/chronologie.html

17. Un courant d'air traverse en effet ces terres et participe de la régulation du climat urbain de la cuvette où est installé le centre-ville de Stuttgart.

18. http://ec.europa.eu/eurostat/statistics-explained/index.php/Farm_structure_statistics/fr

\section{RÉSUMÉS}

«L'agriculture dans la ville, pour la ville» est le slogan adopté par le Reyerhof, une ferme en polyculture-élevage biodynamique. Ce slogan traduit certes un argument commercial mais aussi un état de fait. Les parcelles que celle-ci travaille s'inscrivent en effet dans le territoire urbanisé de Stuttgart. Elles occupent la lisière des quartiers ou alors s'intercalent entre eux. L'auteur, qui est paysagiste et dessinateur, convie à un voyage en terres urbaines cultivées à la rencontre des paysages agricoles et de ceux qui les cultivent. Saisir, décrire et représenter les paysages de cette exploitation agricole métropolitaine est le sens donné à cette contribution en rassemblant un peu de matières premières à ce qui pourrait potentiellement devenir un travail plus large de recherche et de projet.

„Landwirtschaft in der Stadt für die Stadt" : so lautet der Werbespruch, den der Reyerhof, ein biologisch-dynamischer Gemischtbetrieb, gewählt hat. Dieser Spruch dient der Werbung und drückt zugleich einen tatsächlichen Sachverhalt aus. Denn die vom Reyerhof bewirtschafteten Parzellen liegen inmitten des Stuttgarter Stadtgebiets. Sie grenzen direkt an die bebaute Fläche an oder fügen sich dazwischen ein. Der Autor - Landschaftsarchitekt und Zeichner - lädt zu einer Erkundung der landwirtschaftlich genutzten Flächen im Stadtgebiet ein, um die Agrarlandschaften und diejenigen, die diese Flächen bewirtschaften, kennenzulernen. In diesem Beitrag soll es darum gehen, einige landschaftliche Gegebenheiten dieses Landwirtschaftsbetriebs in der Großstadt zu erfassen, zu beschreiben und darzustellen. Das hier zusammengetragene Material könnte Ausgangspunkt für eine breiter angelegte Untersuchung sein.

\section{INDEX}

Schlüsselwörter : ländlicher Raum, städtischer Raum, Landwirtschaft, Großstadt, Landwirt, Verbraucher, Direktvermarktung, Landschaftsarchitekt, Stuttgart

Mots-clés : paysage agricole et urbain, agriculture, métropole, agriculteur, consommateur, vente directe, paysagiste, Stuttgart 


\section{AUTEUR}

\section{DOMINIQUE HENRY}

Paysagiste DPLG et docteur en géographie, Dominique Henry est maître-assistant à l'École nationale supérieure d'architecture et de paysage de Lille, chercheur à l'UMR 5319 Passages et travaille, en indépendant, dans Le champ d'à côté (bureau mobile de paysage). Ses travaux et ses recherches s'intéressent aux paysages liés au travail de la terre, de l'échelle du jardin à celle plus vaste des territoires ruraux et agricoles.

lechampdacote[at]gmail[dot]com 